Network Working Group

Request for Comments: 1315
C. Brown

Wellfleet Communications, Inc.

F. Baker

Advanced Computer Communications

C. Carvalho

Advanced Computer Communications

April 1992

\title{
Management Information Base for Frame Relay DTEs
}

Status of this Memo

This RFC specifies an IAB standards track protocol for the Internet community, and requests discussion and suggestions for improvements. Please refer to the current edition of the "IAB Official Protocol

Standards" for the standardization state and status of this protocol. Distribution of this memo is unlimited.

\section{Abstract}

This memo defines a portion of the Management Information Base (MIB) for use with network management protocols in TCP/IP-based internets. In particular, it defines objects for managing Frame Relay.

Table of Contents

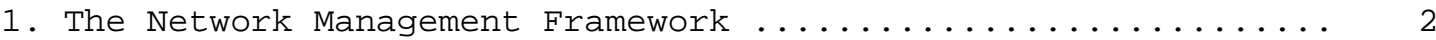

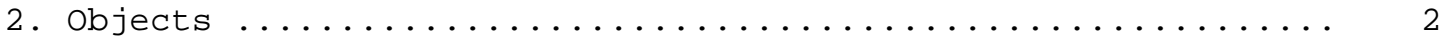

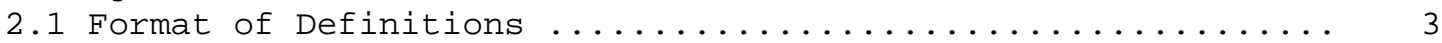

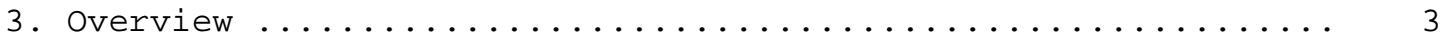

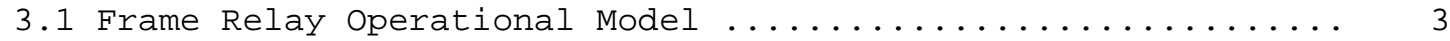

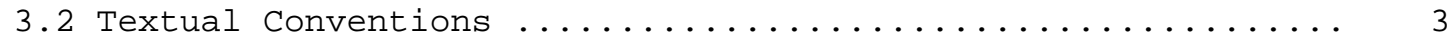

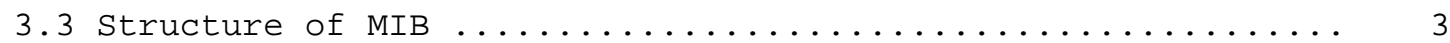

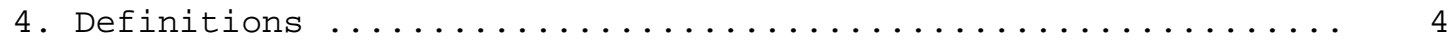

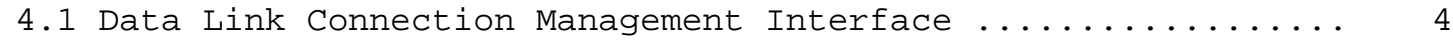

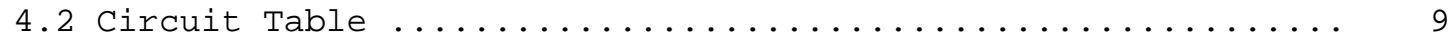

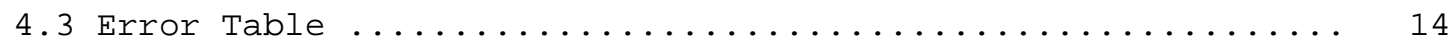

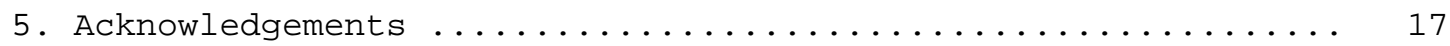

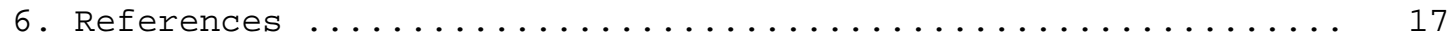

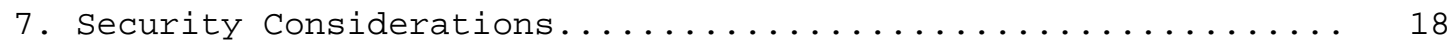

8. Authors' Addresses................................ 19 
1. The Network Management Framework

The Internet-standard Network Management Framework consists of three components. They are:

RFC 1155 which defines the SMI, the mechanisms used for describing and naming objects for the purpose of management. RFC 1212 defines a more concise description mechanism, which is wholly consistent with the SMI.

RFC 1156 which defines MIB-I, the core set of managed objects for the Internet suite of protocols. RFC 1213 defines MIB-II, an evolution of MIB-I based on implementation experience and new operational requirements.

RFC 1157 which defines the SNMP, the protocol used for network access to managed objects.

The Framework permits new objects to be defined for the purpose of experimentation and evaluation.

\section{Objects}

Managed objects are accessed via a virtual information store, termed the Management Information Base or MIB. Objects in the MIB are defined using the subset of Abstract Syntax Notation One (ASN.1) [7] defined in the SMI. In particular, each object has a name, a syntax, and an encoding. The name is an object identifier, an administratively assigned name, which specifies an object type. The object type together with an object instance serves to uniquely identify a specific instantiation of the object. For human convenience, we often use a textual string, termed the OBJECT DESCRIPTOR, to also refer to the object type.

The syntax of an object type defines the abstract data structure corresponding to that object type. The ASN.1 language is used for this purpose. However, the SMI [3] purposely restricts the ASN.1 constructs which may be used. These restrictions are explicitly made for simplicity.

The encoding of an object type is simply how that object type is represented using the object type's syntax. Implicitly tied to the notion of an object type's syntax and encoding is how the object type is represented when being transmitted on the network.

The SMI specifies the use of the basic encoding rules of ASN.1 [8], subject to the additional requirements imposed by the SNMP. 


\subsection{Format of Definitions}

Section 4 contains contains the specification of all object types contained in this MIB module. The object types are defined using the conventions defined in the SMI, as amended by the extensions specified in $[9,10]$.

3. Overview

\subsection{Frame Relay Operational Model}

For the purposes of understanding this document, Frame Relay is viewed as a multi-access media, not as a group of point-to-point connections. This model proposes that Frame Relay is a single interface to the network (physical connection) with many destinations or neighbors (virtual connections). This view enables a network manager the ability to group all virtual connections with their corresponding physical connection thereby allowing simpler diagnostics and trouble shooting.

\subsection{Textual Conventions}

Several new data types are introduced as a textual convention in this MIB document. These textual conventions enhance the readability of the specification and can ease comparison with other specifications if appropriate. It should be noted that the introduction of the these textual conventions has no effect on either the syntax nor the semantics of any managed objects. The use of these is merely an artifact of the explanatory method used. Objects defined in terms of one of these methods are always encoded by means of the rules that define the primitive type. Hence, no changes to the SMI or the SNMP are necessary to accommodate these textual conventions which are adopted merely for the convenience of readers and writers in pursuit of the elusive goal of clear, concise, and unambiguous MIB documents.

The new data types are Index and DLCI. Index refers to the range 1...ifNumber, and is used to establish the correspondence between ifEntries and Frame Relay Interfaces. DLCI refers to the range 0 ..DLCINumber, and is used to refer to the valid Data Link Connection Indices. DLCINumber is, by definition, the largest possible DLCI value possible under the configured Q.922 Address Format.

\subsection{Structure of MIB}

The MIB is composed of three groups, one defining the Data Link Connection Management Interface (DLCMI), one describing the Circuits, and a third describing errors. 
During normal operation, Frame Relay virtual circuits will be added, deleted and change availability. The occurrence of such changes is of interest to the network manager and therefore, one trap is defined, intended to be corollary to the SNMP "Link Up" and "Link Down" traps.

4. Definitions

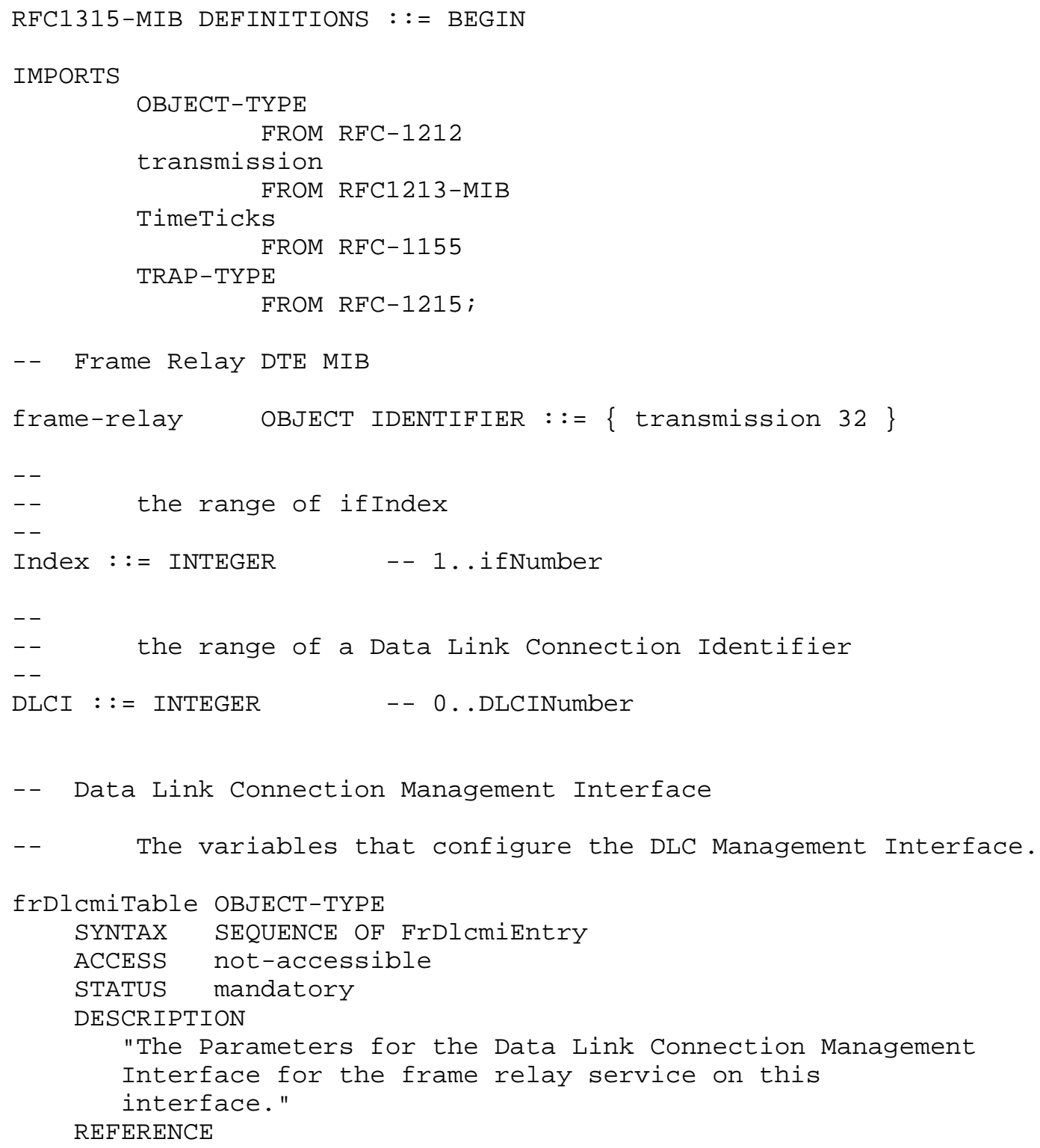




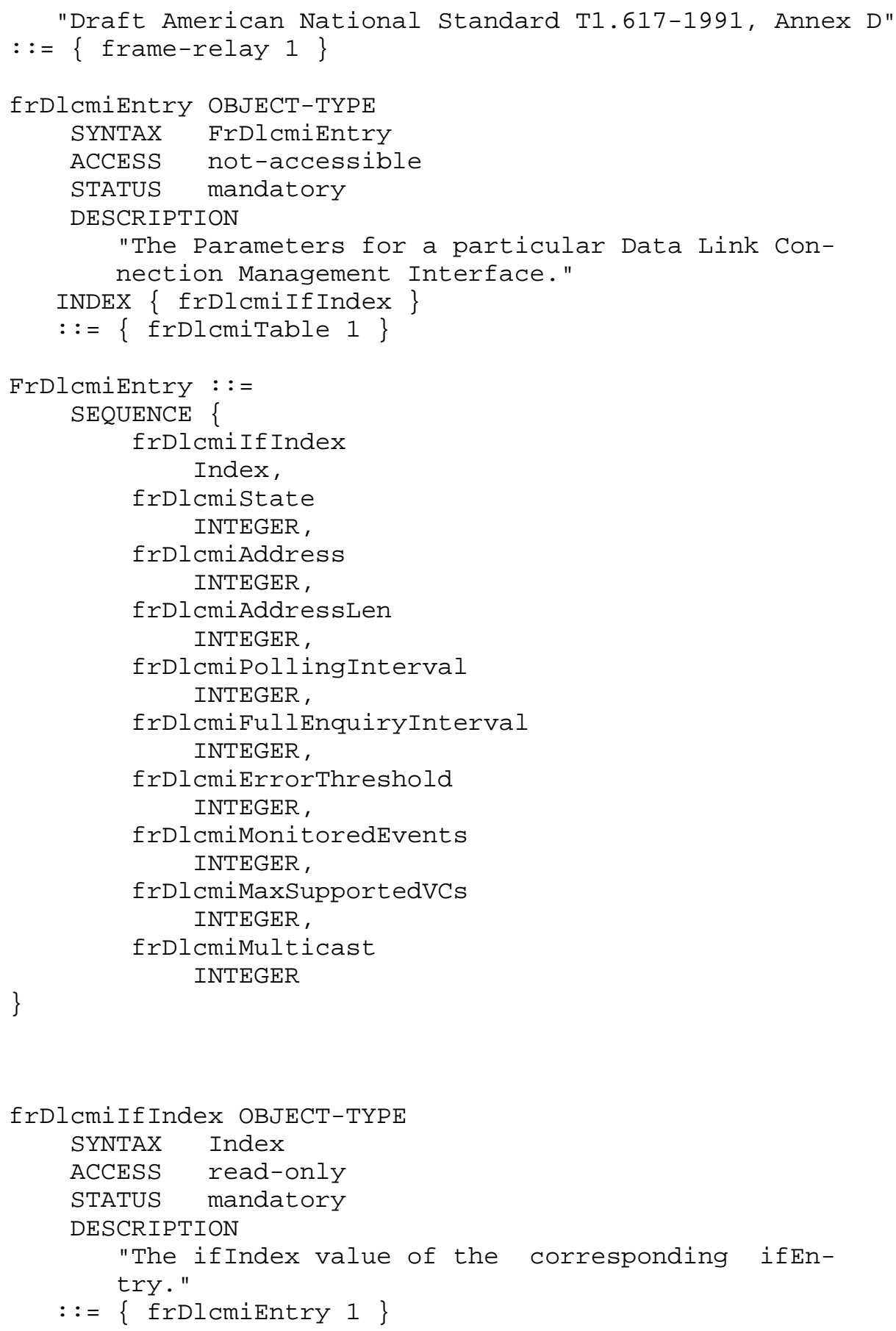

Brown, Baker \& Carvalho 


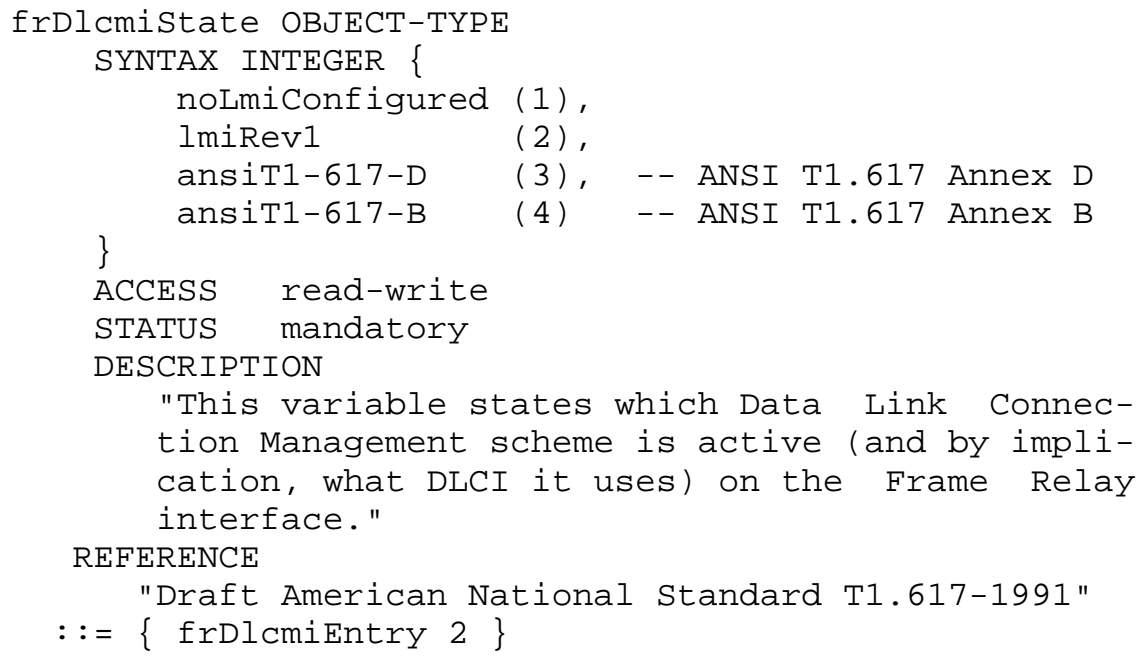

Brown, Baker \& Carvalho 


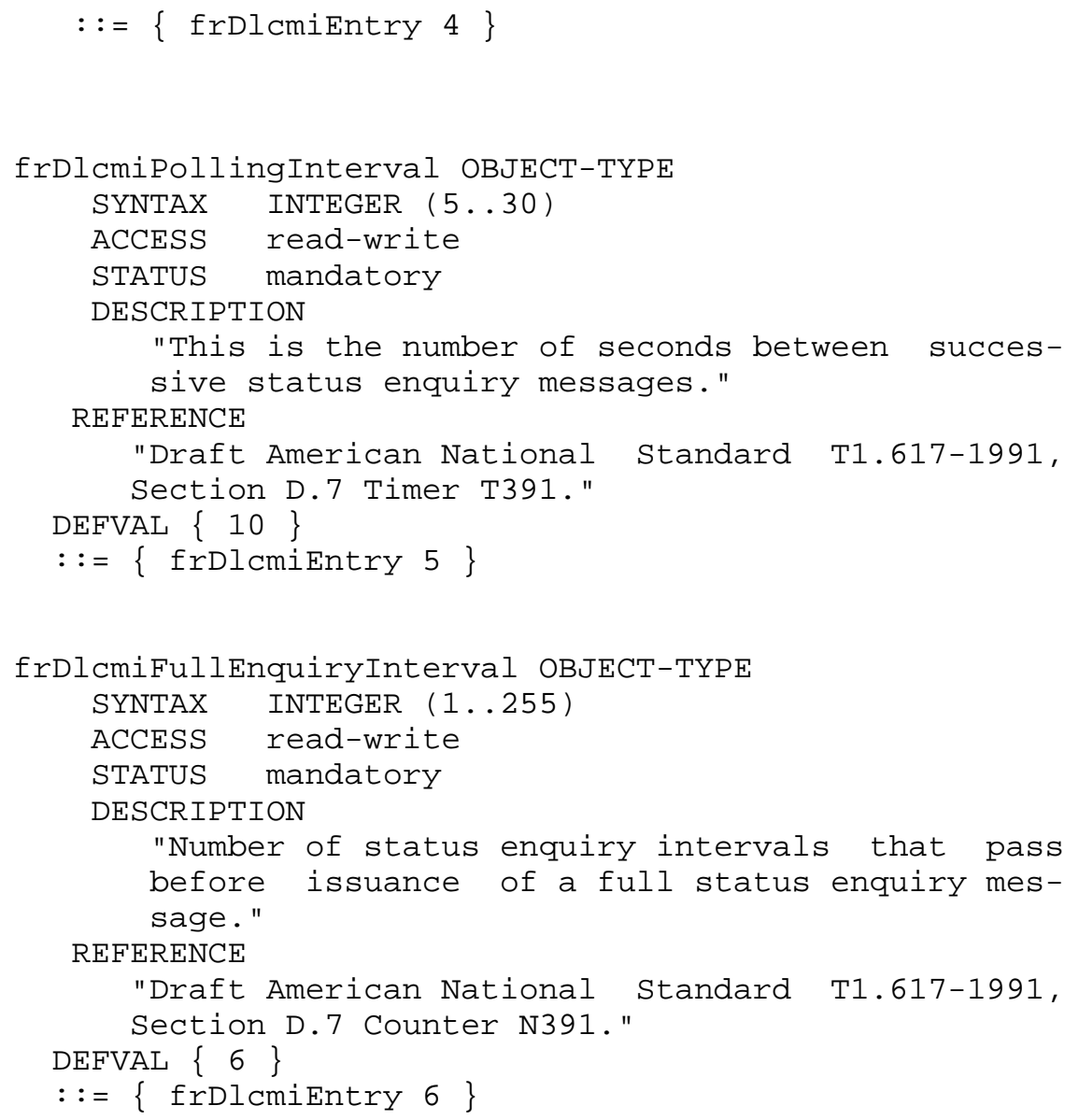

Brown, Baker \& Carvalho 


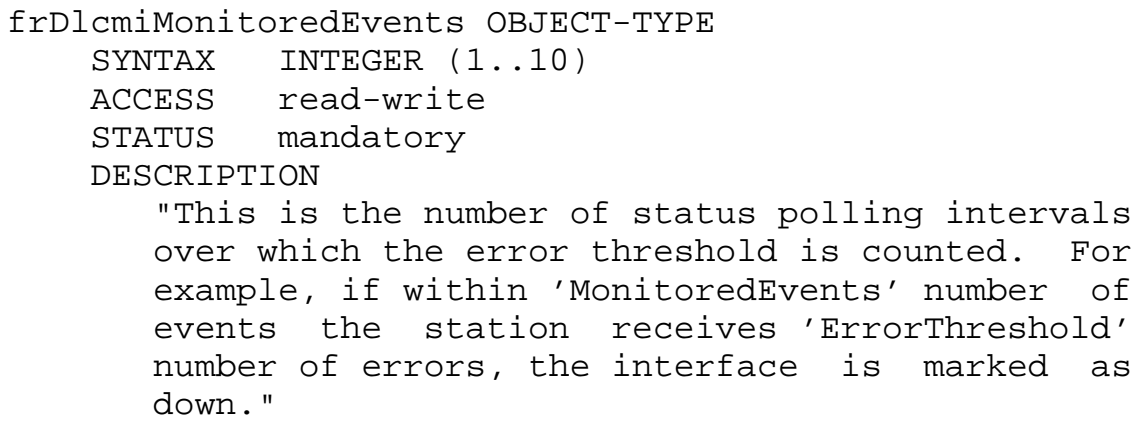

"The maximum number of Virtual Circuits allowed for this interface. Usually dictated by the Frame Relay network.

In response to a $\mathrm{SET}$, if a value less than zero or higher than the agent's maximal capability is configured, the agent should respond badValue" 
-- A Frame Relay service is a multiplexing service. Data

-- Link Connection Identifiers enumerate virtual circuits

-- (permanent or dynamic) which are layered onto the underlying

-- circuit, represented by ifEntry. Therefore, each of the entries

-- in the Standard MIB's Interface Table with an IfType of

-- Frame Relay represents a Q.922 interface. Zero or more

-- virtual circuits are layered onto this interface and provide

-- interconnection with various remote destinations.

-- Each such virtual circuit is represented by an entry in the

-- circuit table.

-- Circuit Table

-- The table describing the use of the DLCIs attached to

-- each Frame Relay Interface.

frCircuitTable OBJECT-TYPE

SYNTAX SEQUENCE OF FrCircuitEntry

ACCESS not-accessible

STATUS mandatory

DESCRIPTION

"A table containing information about specific Data

Link Connection Identifiers and corresponding virtual

circuits."

$::=\{$ frame-relay 2$\}$

frCircuitentry OBJECT-TYPE

SYNTAX FrCircuitEntry

ACCESS not-accessible

STATUS mandatory

DESCRIPTION

"The information regarding a single Data Link Connection Identifier."

INDEX \{ frCircuitIfIndex, frCircuitDlci \}

$::=\{$ frCircuitTable 1$\}$

FrCircuitentry : := SEQUENCE \{

frCircuitIfIndex

Index,

frCircuitDlci

DLCI,

frCircuitstate

INTEGER,

frCircuitReceivedFECNs

Counter,

frCircuitReceivedBECNs 


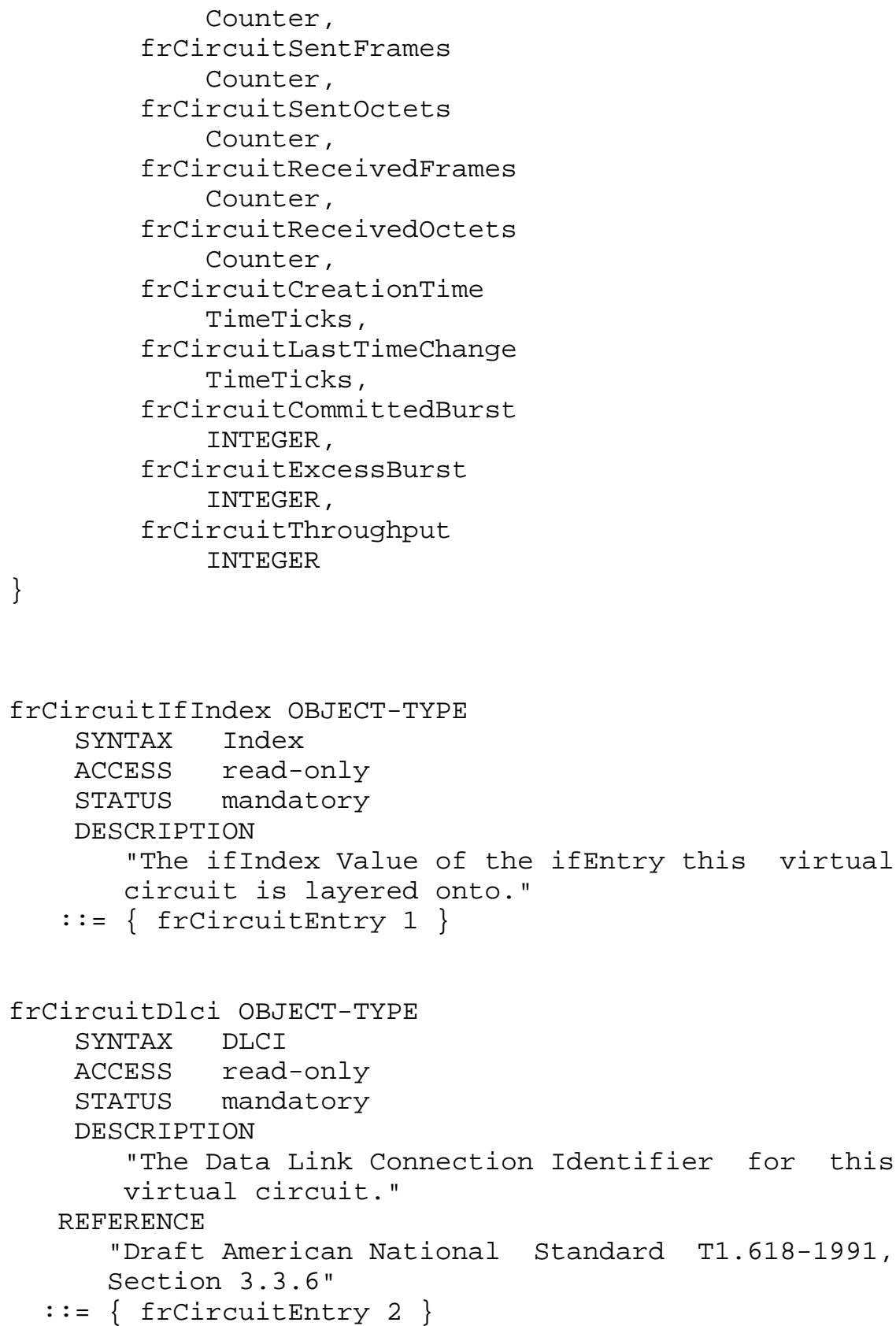

Brown, Baker \& Carvalho 


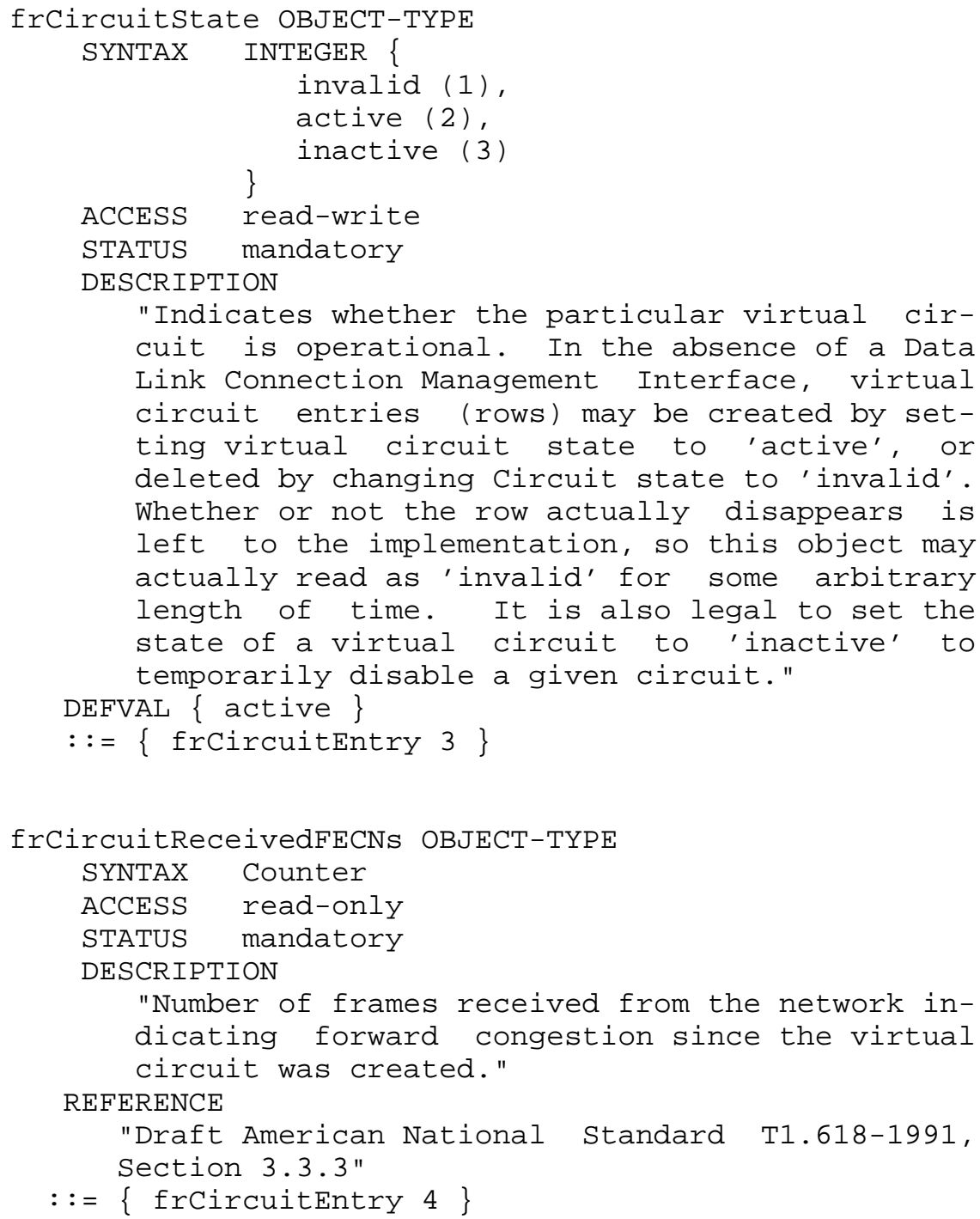

"Number of frames received from the network indicating backward congestion since the virtual circuit was created." 


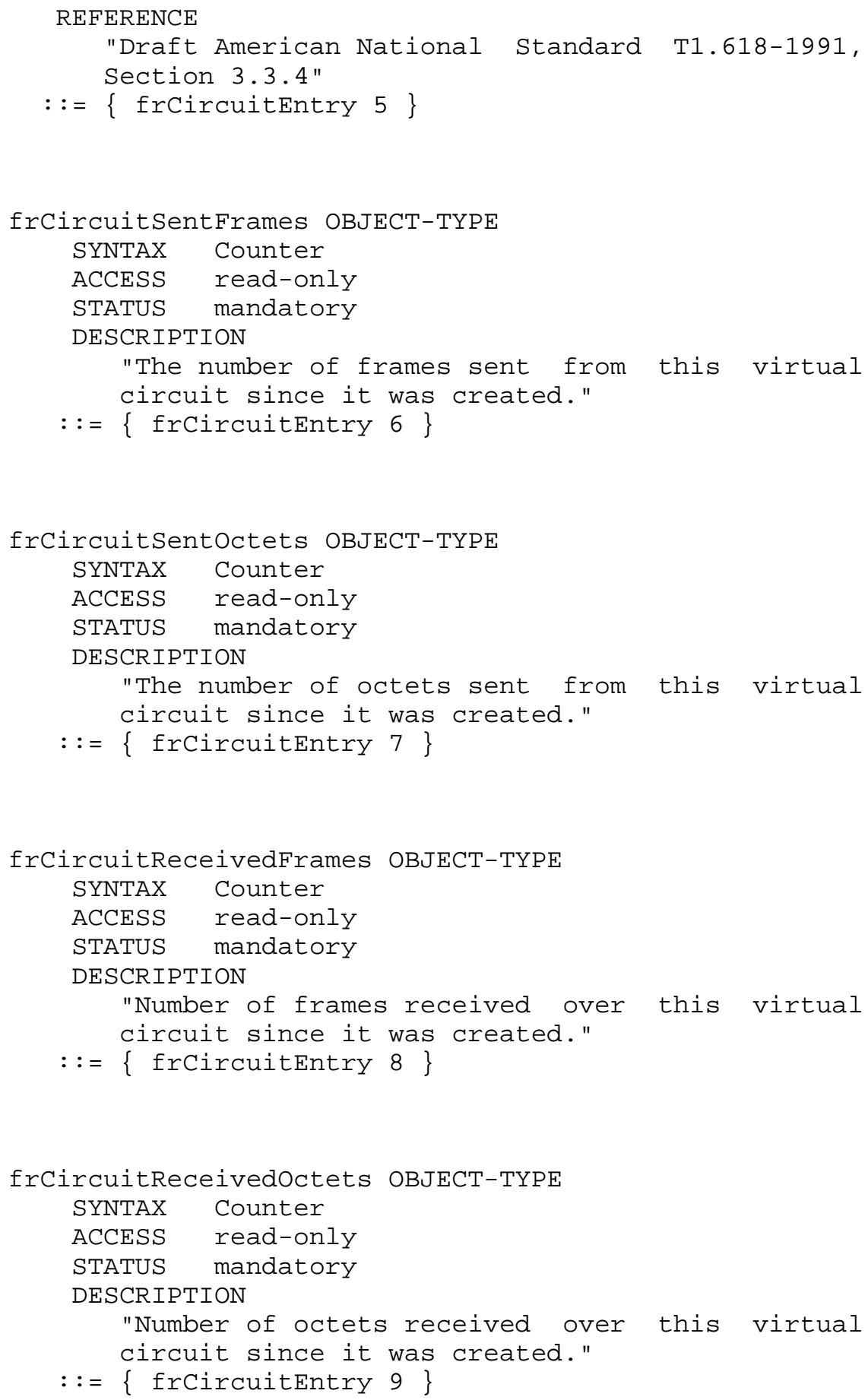

Brown, Baker \& Carvalho 
tempt to deliver over the measurement interval.

By default, if not configured when creating the entry, the Excess Information Burst Size is set to the value of ifspeed." REFERENCE

"Draft American National Standard T1.617-1991, Section 6.5.19" $::=\{$ frCircuitentry 13$\}$

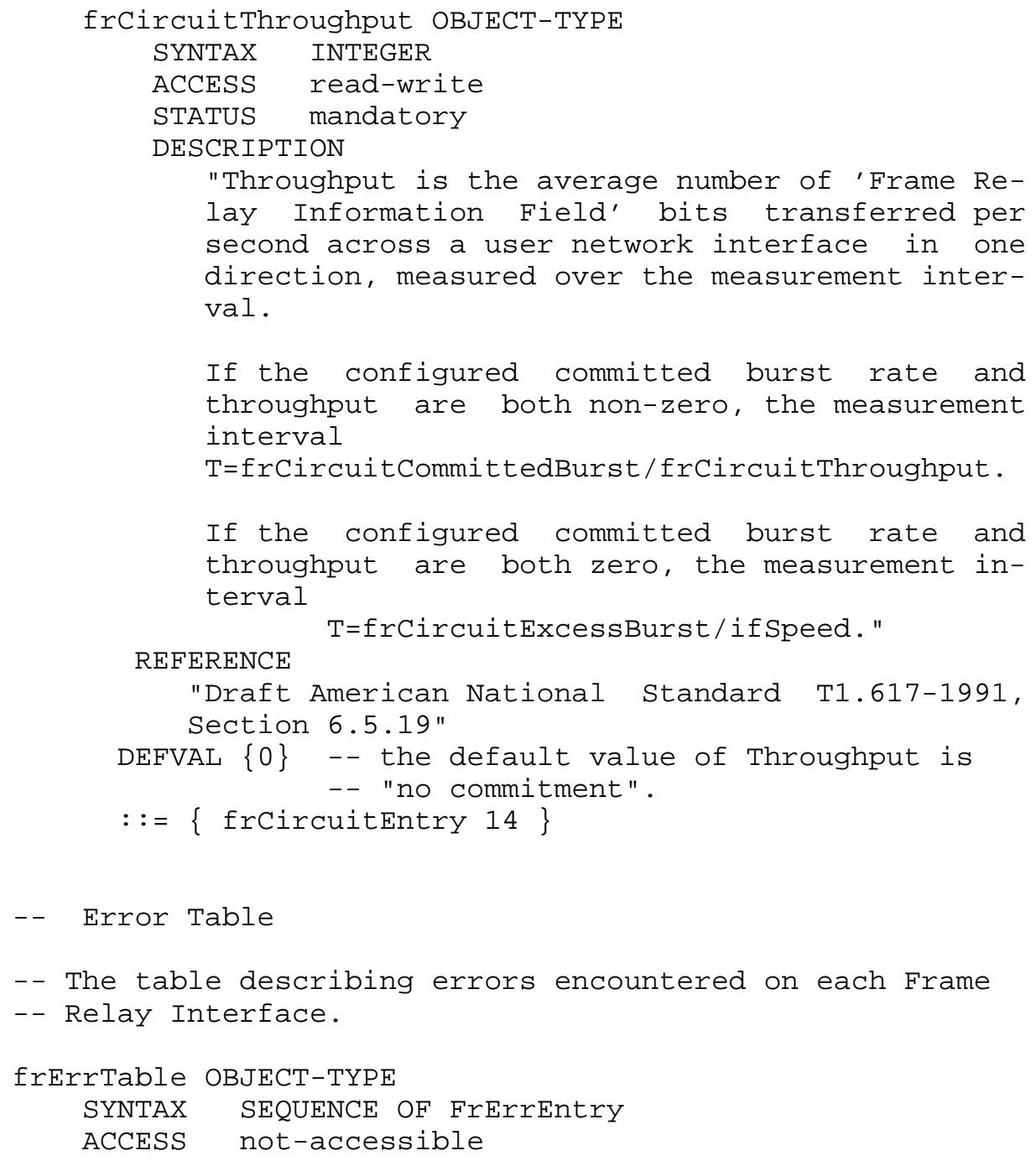




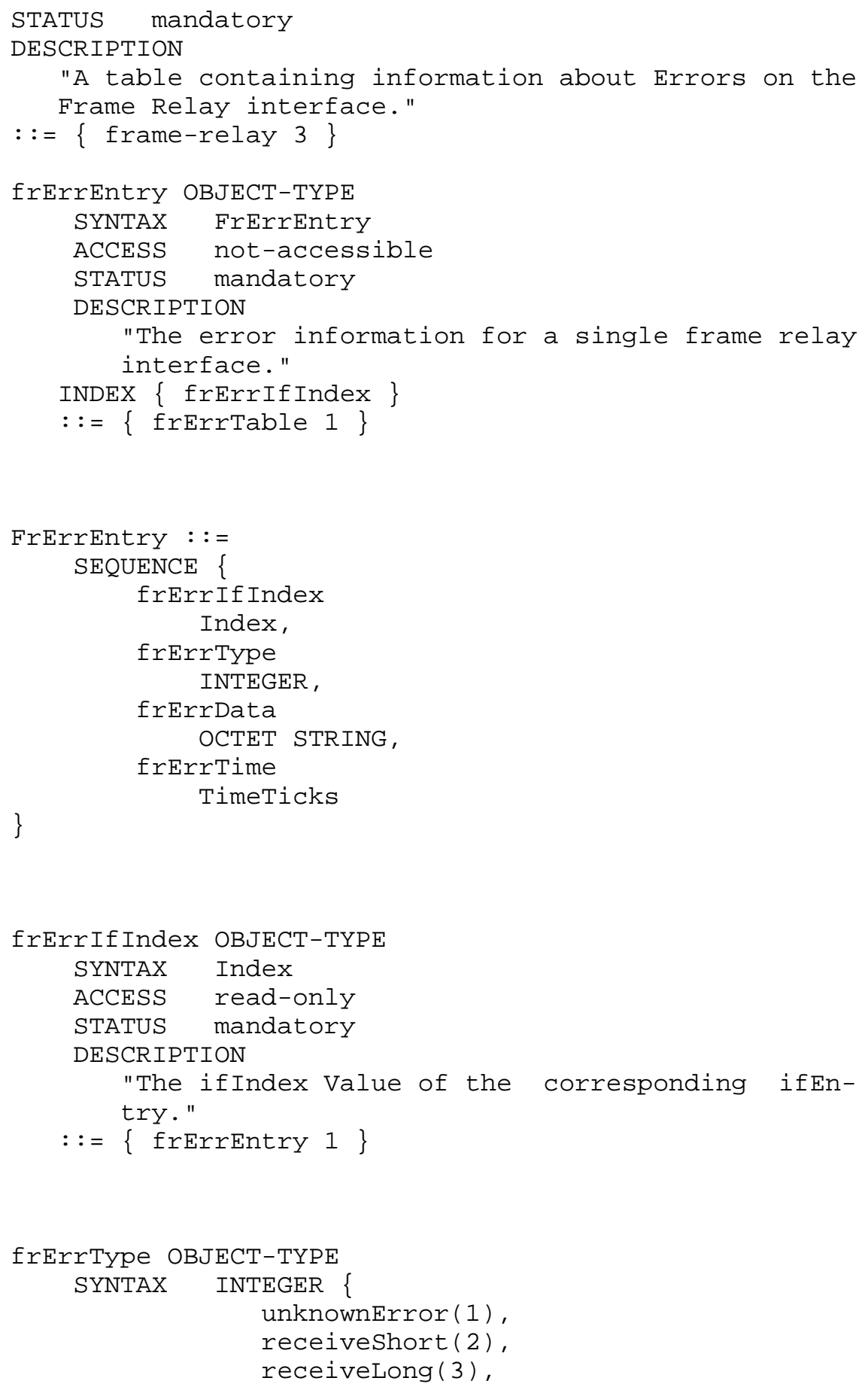

Brown, Baker \& Carvalho 


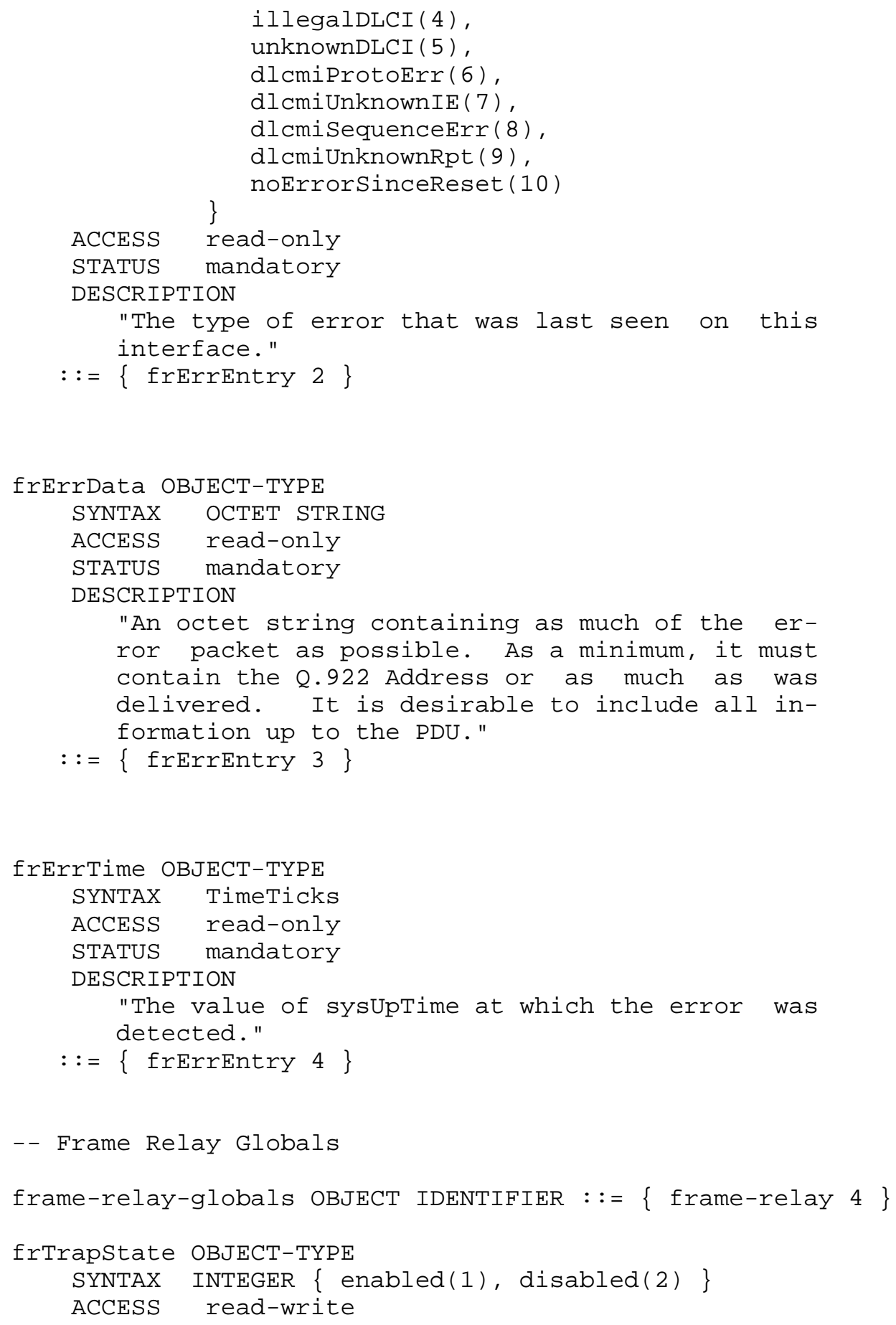




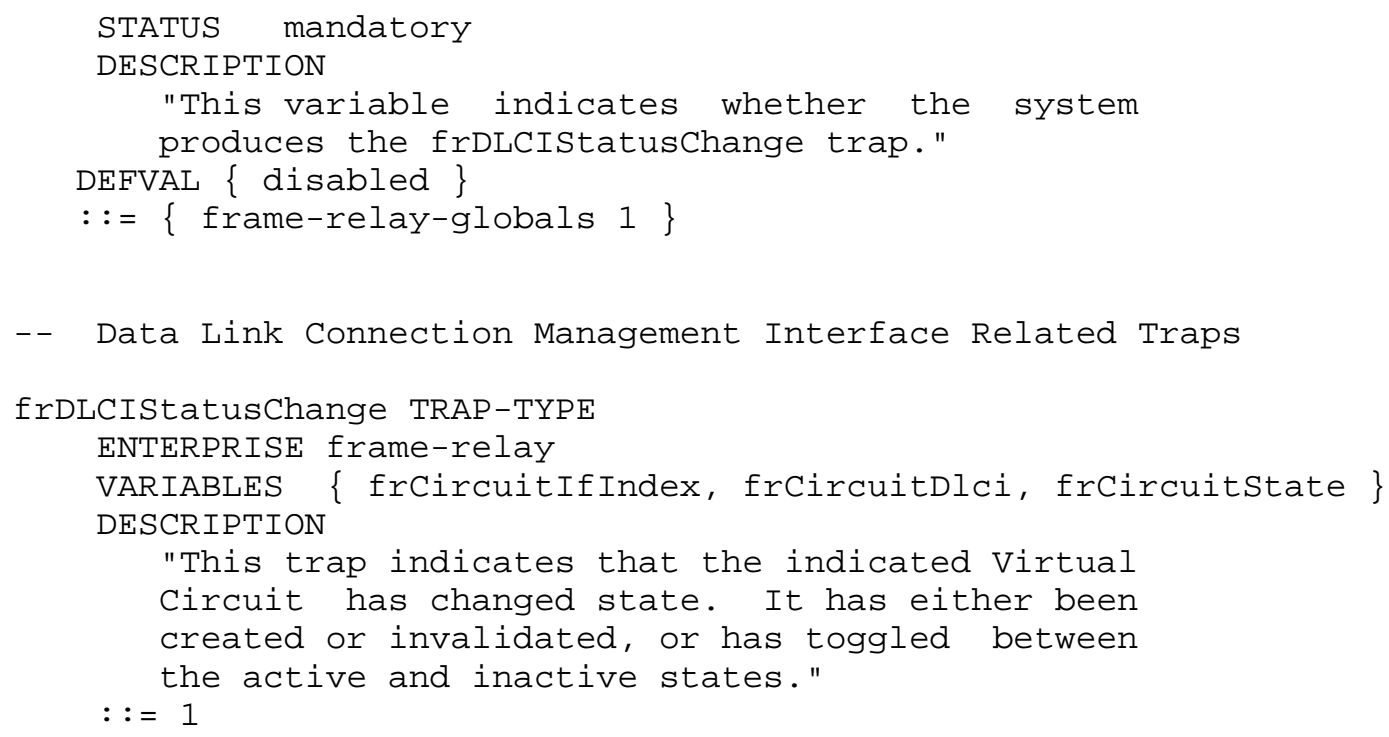

END

5. Acknowledgements

This document was produced by the IP Over Large Public Data Networks (IPLPDN) Working Group.

The following people provided additional comments and suggestions: Art Berggreen of Advanced Computer Communications, and Jim Philippou of Xyplex Communications.

6. References

[1] Cerf, V., "IAB Recommendations for the Development of Internet Network Management Standards", RFC 1052, NRI, April 1988.

[2] Cerf, V., "Report of the Second Ad Hoc Network Management Review Group", RFC 1109, NRI, August 1989.

[3] Rose M., and K. McCloghrie, "Structure and Identification of Management Information for TCP/IP-based internets", RFC 1155, Performance Systems International, Hughes LAN Systems, May 1990.

[4] McCloghrie K., and M. Rose, "Management Information Base for Network Management of TCP/IP-based internets", RFC 1156, Hughes LAN Systems, Performance Systems International, May 1990. 
[5] Case, J., Fedor, M., Schoffstall, M., and J. Davin, "Simple Network Management Protocol", RFC 1157, SNMP Research, Performance Systems International, Performance Systems International, MIT Laboratory for Computer Science, May 1990.

[6] McCloghrie K., and M. Rose, Editors, "Management Information Base for Network Management of TCP/IP-based internets", RFC 1213, Performance Systems International, March 1991.

[7] Information processing systems - Open systems Interconnection Specification of Abstract Syntax Notation One (ASN.1), International Organization for Standardization, International Standard 8824, December 1987.

[8] Information processing systems - Open Systems Interconnection Specification of Basic Encoding Rules for Abstract Notation One (ASN.1), International Organization for Standardization, International Standard 8825, December 1987.

[9] Rose, M., and K. McCloghrie, Editors, "Concise MIB Definitions", RFC 1212, Performance Systems International, Hughes LAN Systems, March 1991.

[10] Rose, M., Editor, "A Convention for Defining Traps for use with the SNMP", RFC 1215, Performance Systems International, March 1991 .

7. Security Considerations

Security issues are not discussed in this memo. 
8. Authors' Addresses

Caralyn Brown

Wellfleet Communications, Inc.

15 Crosby Drive

Bedford, Massachusetts 01730

Phone: (617) 275-2400

EMail: cbrown@wellfleet.com

Fred Baker

Advanced Computer Communications

315 Bollay Drive

Sannta Barbara, California 93117

Phone: (805) 685-4455

EMail: fbakeraacc.com

Charles Carvalho

Advanced Computer Communications

315 Bollay Drive

Sannta Barbara, California 93117

Phone: (805) 685-4455

EMail: charles@acc.com 